\title{
Estimativas de parâmetros genéticos de características reprodutivas de touros Nelore, de dois e três anos de idade
}

[Genetic parameter estimates for reproductive traits in young Nelore bulls]

\author{
J.C. Dias $^{1,3}$, V.J. Andrade ${ }^{2 *}$, A.B. Fridrich ${ }^{1,3}$, D.F. Salvador ${ }^{1,3}$, V.R. Vale Filho ${ }^{2}$, \\ A.B. Corrêa ${ }^{1,3}$, M.A. Silva ${ }^{2}$ \\ ${ }^{1}$ Aluno de pós-graduação - EVUFMG \\ ${ }^{2}$ Escola de Veterinária - UFMG \\ Caixa Postal 567 \\ 30123-970 - Belo Horizonte, MG \\ ${ }^{3}$ Bolsista do CNPq
}

\begin{abstract}
RESUMO
Estimaram-se a herdabilidade e a correlação genética entre características ponderais e reprodutivas de 579 touros da raça Nelore, de 19 a 39 meses de idade. As características reprodutivas avaliadas foram: circunferência escrotal (CE); consistência, volume, forma, comprimento e largura dos testículos; motilidade e vigor dos espermatozóides; defeitos espermáticos maiores, menores e totais; e classificação andrológica por pontos (CAP). As características foram analisadas pelo método de máxima verossimilhança restrita, com algoritmos livres de derivadas, sob modelo animal, com inclusão da matriz de numeradores dos coeficientes de parentesco entre os animais e seus ascendentes, utilizando o programa MTDFREML. As estimativas de herdabilidade para consistência testicular, motilidade e vigor espermáticos e defeitos espermáticos maiores, menores e totais e CAP foram, respectivamente, 0,46; 0,$10 ; 0,08 ; 0,16 ; 0,09 ; 0,11$ e 0,10 . As correlações genéticas entre $\mathrm{CE}$ e: peso, volume testicular, motilidade e vigor espermáticos, defeitos espermáticos menores, defeitos totais e CAP foram, respectivamente, 0,$72 ; 0,99 ; 0,72 ; 0,60 ;-0,67 ;-0,12$ e 0,64 . As correlações genéticas entre CAP e: peso, volume testicular, defeitos espermáticos maiores e defeitos totais foram, respectivamente, 0,$19 ; 0,71$; $-0,47$ e $-0,58$. Os resultados sugerem compatibilidade de crescimento corporal e fertilidade nos programas de seleção de reprodutores Nelore.
\end{abstract}

Palavras-chave: biometria testicular, característica seminal, correlação genética, herdabilidade, Nelore

\begin{abstract}
Heritabilities and genetic correlations between performance and reproductive traits were estimated using Multiple Trait Derivative-Free Restricted Maximum Likelihood methodology in 579 pasture-raised Nelore bulls that were 19 to 39 months of age. Traits were breeding soundness evaluation (BSE), scrotal circumference (SC), testicular consistency (TC), testicular volume (TV), testicular shape (TS), length and width of right and left testicles, and semen traits including motility (Mot), vigor (Vig), major (MD) sperm defects, minor $(\mathrm{mD})$ sperm defects and total (TD) sperm defects. Heritability estimates were 0.46; 0.10; 0.08 ; 0.16 ; 0.09; 0.11 and 0.10 for TC, Mot, Vig, $M D, m D, T D$ and BSE, respectively. Genetic correlations between SC and: body weight (BW), TV, Mot, Vig, $m D$, TD and BSE were 0.72; 0.99; 0.72; $0.60 ;-0.67 ;-0.12$ and 0.64, respectively, showing favorable associations between SC and the other traits. Genetic correlations between BSE and: BW, TV, MD and TD were 0.19; $0.71 ;-0.47$ and -0.58 ,
\end{abstract}

Recebido em 12 de maio de 2004

Aceito em 8 de novembro de 2005

*Autor para correspondência (corresponding author)

E-mail: vejoan@dedalus.lcc.ufmg.br 
respectively. Results suggest that selection for body weight in Nelore bulls should not hamper fertility, nor should selection for reproductive traits hamper growth.

Keywords: testicular biometry, seminal traits, genetic correlation, heritability, Nelore

\section{INTRODUÇÃO}

O aumento da produtividade dos rebanhos bovinos pode ser obtido a partir da identificação e multiplicação de genótipos superiores e por meio de adequação das condições ambientes ao processo produtivo. A identificação de efeitos ambientes relacionados à eficiência reprodutiva é importante por possibilitar alterações, quando necessárias, do manejo dos animais, visando à maior produtividade. Além disso, permite ajustamentos e correções para os efeitos ambientes necessários à obtenção de parâmetros genéticos e estimativas do valor genético dos animais e, dessa forma, possibilita o correto estabelecimento de planos de melhoramento (Gressler et al., 2000).

A eficiência reprodutiva pode ser considerada uma das mais importantes características econômicas, devendo receber especial atenção dos criadores de gado de corte. Rebanhos detentores de elevada precocidade sexual e fertilidade permitem maior intensidade seletiva e, conseqüentemente, progressos genéticos mais elevados e maior lucratividade (Andrade et al., 2001).

A reprodução é um processo complexo, e a seleção direta para características ligadas à reprodução é, muitas vezes, difícil de ser aplicada, tornando-se necessário identificar características reprodutivas que sejam facilmente medidas, que apresentem variabilidade e que sejam geneticamente correlacionadas aos eventos reprodutivos (Bergmann, 1999).

Dentre as características reprodutivas, tem-se recomendado incluir a circunferência escrotal nos programas de seleção dos touros de corte, por estar geneticamente associada, de maneira favorável, às características seminais, ponderais e às características reprodutivas das fêmeas, sugerindo que o melhoramento da fertilidade, tanto dos machos quanto das fêmeas, poderá se dar por meio da seleção indireta baseada na circunferência escrotal (Bergmann et al., 1996; Bergmann, 1999).
Existem poucos relatos na literatura a respeito das associações genéticas entre características ponderais, medidas testiculares e características seminais para touros de raças zebuínas (Bos taurus indicus) no Brasil. Entretanto, alguns estudos (Vale Filho et al., 1999; Bergmann et al., 1996; Quirino et al., 1998; Martinez et al., 2000; Dias et al., 2003) têm demonstrado resposta correlacionada em características seminais e ponderais quando se seleciona para circunferência escrotal.

O objetivo do presente estudo foi o de estimar correlações genética, e herdabilidades de características reprodutivas e produtivas de touros da raça Nelore, criados extensivamente.

\section{MATERIAL E MÉTODOS}

Os dados referem-se a características reprodutivas e produtivas de 579 touros da raça Nelore, com idade variando entre 19 e 39 meses, oriundos de um rebanho registrado, de propriedade da Agropecuária Nove de Ouro, localizada no município de Miranda - MS, avaliados entre os anos de 2000 e 2002.

Estudaram-se características de biometria testicular - circunferência escrotal (CE); consistência (CON), volume (VOL) e forma dos testículos (FOR); comprimento do testículo esquerdo (CTE) e do testículo direito (CTD); largura do testículo esquerdo (LTE) e do testículo direito (LTD) - características físicas motilidade (MOT) e vigor - e características morfológicas - defeitos espermáticos maiores (DM), menores (DMe) e totais (DT) do sêmen (Tab. 1), conforme descrito pelo Colégio Brasileiro de Reprodução Animal (Manual... 1998). Estudou-se, ainda, a classificação andrológica por pontos (CAP) (Tab. 1), segundo Vale Filho (1989), para se estabelecerem os coeficientes de herdabilidade das características e as correlações genéticas e fenotípicas entre essas características e o peso de animais Nelore, criados extensivamente. 
Tabela 1. Número de animais e médias do peso e das características reprodutivas de touros Nelore criados extensivamente, segundo a faixa etária

\begin{tabular}{|c|c|c|c|c|c|c|c|c|c|c|c|}
\hline $\begin{array}{l}\text { Idade } \\
\text { (meses) }\end{array}$ & $\mathrm{n}$ & $\begin{array}{l}\text { Peso } \\
(\mathrm{kg})\end{array}$ & $\begin{array}{c}\mathrm{CE} \\
(\mathrm{cm})\end{array}$ & $\begin{array}{c}\text { MOT } \\
(\%) \\
\end{array}$ & $\begin{array}{l}\text { Vigor } \\
(1-5)\end{array}$ & $\begin{array}{l}\text { VOL } \\
(\mathrm{cm} 3) \\
\end{array}$ & $\begin{array}{l}\text { CON } \\
(1-5) \\
\end{array}$ & $\begin{array}{l}\text { DM } \\
(\%)\end{array}$ & $\begin{array}{c}\text { DMe } \\
(\%) \\
\end{array}$ & $\begin{array}{l}\text { DT } \\
(\%) \\
\end{array}$ & $\begin{array}{c}\text { CAP } \\
(0-100)\end{array}$ \\
\hline $19-24$ & 396 & $306,4+40,3$ & $26,2 \pm 2,8$ & $48,6 \pm 15,7$ & $2,7 \pm 0,8$ & $415,4 \pm 126,0$ & $4,1 \pm 0,7$ & $22,3 \pm 19,8$ & $11,2 \pm 8,5$ & $33,5 \pm 22,0$ & $46,7 \pm 17,4$ \\
\hline $25-29$ & 44 & $357,0+50,8$ & $28,4 \pm 1,4$ & $48,5 \pm 16,8$ & $2,6 \pm 0,7$ & $498,3 \pm 71,9$ & $4,5 \pm 0,8$ & $17,2 \pm 16,0$ & $11,1 \pm 6,6$ & $28,3 \pm 18,2$ & $44,7 \pm 16,6$ \\
\hline $30-34$ & 73 & $413,2+48,7$ & $30,4 \pm 2,3$ & $48,7 \pm 16,2$ & $2,6 \pm 0,8$ & $626,6 \pm 167,4$ & $4,2 \pm 0,7$ & $23,3 \pm 21,1$ & $9,5 \pm 7,8$ & $32,8 \pm 22,8$ & $46,5 \pm 17,6$ \\
\hline $35-39$ & 66 & $434,5+69,4$ & $30,8 \pm 2,3$ & $51,6 \pm 15,3$ & $2,7 \pm 0,8$ & $678,6 \pm 157,0$ & $3,9 \pm 0,7$ & $28,7 \pm 30,4$ & $11,0 \pm 10,0$ & $39,8 \pm 31,2$ & $43,7 \pm 18,8$ \\
\hline
\end{tabular}

Para as estimativas dos componentes de (co) variância, necessários às estimativas de herdabilidade, dos valores gênicos dos animais e das correlações genéticas e ambientes, foi utilizado o método de máxima verossimilhança restrita, com algoritmos livres de derivadas, sob modelo animal, com inclusão da matriz dos numeradores dos coeficientes de parentesco entre os animais e seus ascendentes. Para tanto, utilizou-se o programa Multiple Trait DerivativeFree Restricted Maximum Likelihood (MTDFREML), descrito por Boldman et al. (1995).

As estimativas dos componentes de variância e dos coeficientes de herdabilidade foram obtidas a partir de análises com características únicas para cada uma das variáveis estudadas. As análises bicaracterísticas foram realizadas com o objetivo de se estimar as correlações entre as características reprodutivas avaliadas e o peso corporal.

Os critérios de convergência foram: a) valor de predição da variância do Simplex para localizar o mínimo de $-2 \log \mathrm{L}$ (em que $\mathrm{L}=$ função de máxima verossimilhança) $10^{-6}$ em modelos com duas características; b) não alteração no valor das estimativas do Simplex $-2 \log$ de verossimilhança $10^{-9} \mathrm{em}$ duas repetições sucessivas. A cada ciclo, o programa era reiniciado, usando-se como valores de (co) variância inicial aqueles obtidos na análise anterior.

O modelo utilizado, em notação matricial, foi:

$Y=X b+Z u+e$, em que:

$\mathrm{Y}=$ vetor de observações de características medidas nos indivíduos;

$\mathrm{X}, \mathrm{Z}=$ matrizes de incidências dos efeitos fixos e aleatórios;

$\mathrm{b}=$ vetor de efeitos fixos, incluindo PESO, CON, $\mathrm{CE}$;

$\mathrm{u}=$ vetor dos efeitos aleatórios de valores genético-aditivos diretos do animal;

$\mathrm{e}=$ vetor dos erros aleatórios associados a cada observação.

\section{RESULTADOS E DISCUSSÃO}

A Tab. 2 apresenta as estimativas de componentes de variância genético-aditiva, variância ambiental e herdabilidade, obtidas pela análise unicaracterística, para CON, MOT, vigor, DM, DMe e DT do sêmen e CAP, para touros da raça Nelore.

Tabela 2. Estimativas de componentes de variância genético-aditiva $\left(\sigma_{\mathrm{a}}^{2}\right)$ e ambiente $\left(\sigma_{\mathrm{e}}^{2}\right)$ e herdabilidades $\left(\mathrm{h}^{2}\right)$ de características testiculares, físicas e morfológicas do sêmen e CAP, de touros Nelore

\begin{tabular}{lccc}
\hline Característica & $\sigma_{\mathrm{a}}^{2}$ & $\sigma_{\mathrm{e}}^{2}$ & $\mathrm{~h}^{2} \pm \mathrm{EP}$ \\
\hline CON & 0,26 & 0,31 & $0,46 \pm 0,18$ \\
MOT & 24,36 & 227,48 & $0,10 \pm 0,10$ \\
Vigor & 0,05 & 0,62 & $0,08 \pm 0,09$ \\
DM & 75,48 & 400,14 & $0,16 \pm 0,12$ \\
DMe & 6,82 & 65,66 & $0,09 \pm 0,10$ \\
DT & 62,99 & 492,58 & $0,11 \pm 0,10$ \\
CAP & 29,68 & 279,87 & $0,10 \pm 0,10$ \\
\hline CON = consistência testicular; MOT & $=$ motilidade \\
espermática; DM = defeitos maiores; DMe = defeitos \\
menores; DT = defeitos totais; CAP = classificação \\
andrológica por pontos.
\end{tabular}

A estimativa de herdabilidade da consistência testicular, indicativo da condição espermatogênica, foi alta, 0,46, valor acima daqueles encontrados na literatura pertinente (Quirino, 1999; Coulter et al., 1976).

As estimativas de herdabilidade das características físicas do sêmen apresentaram valores díspares. O valor da herdabilidade para motilidade espermática $(0,10)$ foi próximo, 
porém inferior ao encontrado por Quirino (1999) $(0,15)$; a herdabilidade do vigor espermático $(0,08)$ foi muito mais baixa que as encontradas por Quirino et al. (1998) e Quirino (1999), 0,59 e 0,55 , respectivamente. As condições ambientes e a estrutura dos dados podem, em parte, justificar tais diferenças. As estimativas de herdabilidades dessas características foram de baixa magnitude, porém denotam a presença de variância genético aditiva. $\mathrm{O}$ valor da estimativa de herdabilidade encontrado para motilidade é semelhante ao descrito por Quirino et al. (1998). Knights et al. (1984) e Gipson et al. (1985) também relataram valores de moderado a reduzido para essas características.

As estimativas de herdabilidade das características morfológicas do sêmen foram de baixa a moderada magnitudes (Tab. 2) e indicaram a presença de variância genéticoaditiva, conforme relatou Quirino (1999). Os valores deste estudo foram inferiores aos encontrados por esse mesmo autor.

A estimativa de herdabilidade da CAP foi baixa $(0,10)$, inferior ao valor encontrado por Vale Filho et al. (1999). Esses autores registraram herdabilidade de $0,75 \pm 0,05$, indicando a probabilidade de seleção de touros para alta eficiência reprodutiva com base nessa característica, diferente da estimativa sugerida neste estudo.

$\mathrm{Na}$ Tab. 3, são apresentadas as correlações genéticas entre as várias medidas testiculares (CE, VOL, FOR, CTE, CTD, LTE, LTD e CON) e $\mathrm{o}$ peso. As magnitudes das correlações genéticas entre as características apresentaram-se favoráveis e altas, mostrando base genética comum entre as características, conforme sugeriu Quirino (1999).

Tabela 3. Correlações genéticas entre características biométricas testiculares, em touros Nelore

\begin{tabular}{lcccccccc}
\hline & $\begin{array}{c}\text { CE } \\
(\mathrm{cm})\end{array}$ & $\begin{array}{c}\text { VOL } \\
\left(\mathrm{cm}^{3}\right)\end{array}$ & $\begin{array}{c}\text { CON } \\
(1-5)\end{array}$ & $\begin{array}{c}\text { FOR } \\
(1-5)\end{array}$ & $\begin{array}{l}\text { CTE } \\
(\mathrm{cm})\end{array}$ & $\begin{array}{l}\text { CTD } \\
(\mathrm{cm})\end{array}$ & $\begin{array}{l}\text { LTE } \\
(\mathrm{cm})\end{array}$ & $\begin{array}{l}\text { LTD } \\
(\mathrm{cm})\end{array}$ \\
\hline Peso & 0,72 & 0,75 & 0,28 & 0,80 & 0,44 & 0,32 & 0,84 & 0,84 \\
CE & - & 0,99 & 0,17 & 0,39 & 0,91 & 0,81 & 0,96 & 0,96 \\
VOL & - & - & 0,43 & 0,30 & 0,86 & 0,83 & 0,99 & 0,99 \\
CON & - & - & - & 1,00 & $-0,24$ & $-0,36$ & 0,43 & 0,60 \\
FOR & - & - & - & - & $-0,31$ & $-0,40$ & 0,46 & 0,56 \\
CTE & - & - & - & - & - & 1,00 & 0,75 & 0,79 \\
CTD & - & - & - & - & - & - & 0,71 & 0,75 \\
LTE & - & - & - & - & - & - & - & 1,00 \\
\hline
\end{tabular}

$\mathrm{CE}=$ circunferência escrotal; $\mathrm{VOL}=$ volume testicular; $\mathrm{CON}=$ consistência testicular; $\mathrm{FOR}=$ forma testicular; $\mathrm{CTE}=$ comprimento testículo esquerdo; $\mathrm{CTD}=$ comprimento testículo direito; LTE = largura testículo esquerdo; LTD = largura testículo direito.

A correlação genética favorável e de alta magnitude entre $\mathrm{CE}$ e peso sugere que a primeira é característica adequada para a identificação de touros com maior potencial de ganho de peso, conforme resultados apresentados por Andrade et al. (2001). Valor semelhante de correlação genética entre essas duas características foi registrado por Quirino (1999). Bergmann et al. (1996) encontraram correlações genéticas de menor magnitude para animais de 12 e 18 meses de idade.

As correlações genéticas favoráveis e de alta magnitude entre volume e comprimento e entre volume e largura dos testículos mostram a base genética comum para essas características e a influência direta do comprimento e da largura sobre a massa testicular. As correlações favoráveis e de alta magnitude entre $\mathrm{CE}$ e: VOL, CTE, CTD, LTE e LTD indicam que a CE é uma característica adequada para ser usada nos programas de seleção para predição do tamanho dos testículos em animais da raça Nelore. Andrade et al. (2001) observaram ser a CE mais influenciada pela largura do que pelo comprimento dos testículos, o que poderia comprometer essa associação, em animais de testículos longos.

As correlações genéticas altas e favoráveis entre peso corporal e: CE, VOL, CTE, CTD, LTE e LTD indicam a compatibilidade de seleção para crescimento corporal e fertilidade nos programas de seleção de reprodutores da raça Nelore. De 
modo geral, touros com maior CE apresentam maior massa testicular e maior peso corporal, conforme sugeriu Quirino (1999).
$\mathrm{Na}$ Tab. 4 apresentam-se as correlações genéticas entre peso, características de biometria testicular, características físicas e morfológicas do sêmen e CAP, em touros da raça Nelore.

Tabela 4. Correlações genéticas entre peso, características biométricas testiculares, características físicas e morfológicas do sêmen e CAP, em touros Nelore

\begin{tabular}{lccccccccc}
\hline & $\begin{array}{c}\text { CE } \\
(\mathrm{cm})\end{array}$ & $\begin{array}{c}\text { CON } \\
(1-5)\end{array}$ & $\begin{array}{c}\text { VOL } \\
\left(\mathrm{cm}^{3}\right)\end{array}$ & $\begin{array}{c}\text { MOT } \\
(\%)\end{array}$ & $\begin{array}{c}\text { Vigor } \\
(1-5)\end{array}$ & $\begin{array}{c}\text { DM } \\
(\%)\end{array}$ & $\begin{array}{c}\text { DMe } \\
(\%)\end{array}$ & $\begin{array}{c}\text { DT } \\
(\%)\end{array}$ & $\begin{array}{c}\text { CAP } \\
(0-100)\end{array}$ \\
\hline Peso & $*$ & $*$ & $*$ & 0,22 & 0,02 & $-0,10$ & $-0,19$ & $-0,10$ & 0,19 \\
CE & - & $*$ & $*$ & 0,72 & 0,60 & 0,13 & $-0,67$ & $-0,12$ & 0,64 \\
CONS & - & - & $*$ & $-0,14$ & $-0,79$ & 0,15 & 0,32 & 0,21 & $-0,17$ \\
VOLT & - & - & - & 0,68 & 0,54 & 0,12 & $-0,59$ & $-0,08$ & 0,71 \\
MOTIL & - & - & - & - & 0,65 & 0,00 & $-1,00$ & $-0,71$ & 1,00 \\
Vigor & - & - & - & - & - & $-0,78$ & $-1,00$ & $-1,00$ & 1,00 \\
DM & - & - & - & - & - & - & $-0,39$ & 0,95 & $-0,47$ \\
DMe & - & - & - & - & - & - & - & $-0,08$ & $-0,07$ \\
DT & - & - & - & - & - & - & - & - & $-0,58$ \\
\hline
\end{tabular}

$\mathrm{CE}=$ circunferência escrotal; $\mathrm{CON}=$ consistência testicular; $\mathrm{VOL}=$ volume testicular; MOT = motilidade espermática; DM $=$ defeito maior; $\mathrm{DMe}=$ defeito menor; $\mathrm{DT}=$ defeito total; $\mathrm{CAP}=$ classificação andrológica por pontos. * Ver Tab. 3.

As estimativas de correlação genética entre peso corporal e características físicas do sêmen foram baixas, isto é, entre peso e vigor $(0,02)$ e entre peso e motilidade espermática $(0,22)$, resultados diferentes dos encontrados por Quirino (1999), que relatou correlação de 1,00 entre peso e vigor e de 0,07 entre peso e motilidade. Também aqui as condições ambientes e a estrutura dos dados podem explicar tais diferenças.

Quando consideradas as correlações genéticas entre peso corporal e características morfológicas do sêmen (DM, DMe e DT), percebem-se correlações favoráveis, porém de baixa magnitude. Essas correlações denotam a importância do peso corporal nas características morfológicas do sêmen.

As correlações genéticas entre CE e características físicas do sêmen apresentaram-se favoráveis e de alta magnitude, 0,72 entre CE e motilidade e 0,60 entre $\mathrm{CE}$ e vigor, mostrando a importância da CE nas características físicas estudadas. $\mathrm{Na}$ literatura são encontrados resultados diversos de correlações genéticas entre CE e motilidade, variando de 0,13 a 1,00 (Gipson et al., 1985; Quirino et al., 1998; Quirino, 1999). Os resultados de correlações genéticas entre CE e vigor variam de 0,69 a 0,89 (Quirino et al., 1998; Quirino, 1999). A correlação entre as características físicas do sêmen também foi alta, indicando base genética comum para as características.
As correlações genéticas entre CE e DMe e DT apresentaram valores negativos e, respectivamente, de alta e baixa magnitude, indicando associação favorável entre o desenvolvimento testicular e as características morfológicas do sêmen. Todavia, a correlação genética $(0,13)$ entre CE e DM apresentou valor oposto aos encontrados na literatura, sugerindo possíveis efeitos ambientes e de estrutura dos dados.

As correlações genéticas observadas entre VOL e as outras características estudadas (Tab. 3) foram, na sua maioria, inferiores às encontradas para CE e essas mesmas características, mostrando ser a $\mathrm{CE}$ uma característica mais adequada na seleção de touros da raça Nelore.

A estimativa de correlação genética entre a CAP e o peso foi de baixa magnitude, e as correlações genéticas entre CE, VOL e CAP mostraram a importância do desenvolvimento dos testículos na construção desse índice de classificação de reprodutores. As correlações genéticas negativas e de moderada a alta magnitude entre DM e DT $(-0,47$ e $-0,58$, respectivamente) mostram a importância da morfologia espermática nesse índice de classificação de reprodutores. Não foram encontradas na literatura nacional referências sobre correlações genéticas entre CAP e as características citadas. Estes resultados mostram a importância da inclusão da análise de 
sêmen, juntamente com a CE, na avaliação de touros jovens.

\section{CONCLUSÕES}

As baixas estimativas de herdabilidade das características físicas e morfológicas do sêmen, as correlações genéticas favoráveis entre $\mathrm{CE}$ e essas características e a correlação genética de alta magnitude entre $\mathrm{CE}$ e peso indicam que a $\mathrm{CE}$ é indicada como critério de seleção em programas de melhoramento visando a maior crescimento corporal e a aumento da fertilidade. As correlações genéticas favoráveis e de alta magnitude entre $\mathrm{CE}$ e as demais características de biometria testicular indicam que a CE é uma característica segura de predição do tamanho dos testículos. As correlações genéticas favoráveis e de moderada a alta magnitude entre CAP, peso, $\mathrm{CE}, \mathrm{DM}$ e DT indicam que a CAP pode ser utilizada como critério de seleção de touros Nelore.

\section{REFERÊNCIAS BIBLIOGRÁFICAS}

ANDRADE, V.J.; SALVADOR, D.F.; VALE FILHO, V.R. et al. Perfil andrológico de touros da raça Nelore de dois e três anos de idade, criados extensivamente em condições do estado do Mato Grosso do Sul. Rev. Bras. Reprod. Anim., v.2, p.182-184, 2001.

BERGMANN, J.A.G. Seleção de zebuínos para precocidade sexual. In: SIMPÓSIO DE PRODUÇÃO DE GADO DE CORTE, 1., 1999, Viçosa. Anais... Viçosa: UFV, 1999. p.296.

BERGMANN, J.A.G.; ZAMBORLINI, L.C.; PROCÓPIO, C.S.O. et al. Estimativas de parâmetros genéticos do perímetro escrotal e do peso corporal em animais da raça Nelore. Arq. Bras. Med. Vet. Zootec., v.48, p.69-78, 1996.

BOLDMAN, K.G.; KRIESE, L.; VAN VLECK, L.D. et al. A manual for use for MTDFREML. a set of programs to obtain estimates of variance and covariance [DRAFT]. Lincoln: Departament of Agriculture/Agricultural Research Service, 1995.120p.

COULTER, G.H.; ROUNSAVILLE, T.R.; FOOTE, R.H. Heritability of testicular size and consistency in Holstein bulls. J. Dairy Sci., v.43, p.9-11, 1976.
DIAS, J.C.; SALVADOR, D.F.; ANDRADE, V.J. et al. Seleção andrológica de touros da raça Nelore com três e quatro anos de idade, criados em manejo extensivo. In: REUNIÃO ANUAL DA SOCIEDADE BRASILEIRA DE ZOOTECNIA, 40,, 2003, Santa Maria. Anais... Santa Maria: SBZ, 2003. (CD-ROM)

GIPSON, D.W.; VOGT, D.W.; MASSEY, J.W. et al. Associations of scrotal circumference with semen traits in young bulls. Theriogenology, v.25, p.217-225, 1985.

GRESSLER, S.L.; BERGMANN, J.A.G.; PEREIRA, C.S. et al. Estudo das associações entre perímetro escrotal e características reprodutivas de fêmeas Nelore. Rev. Bras. Zootec., v.29, p.427-437, 2000.

KNIGHTS, S.A.; BAKER, R.L.; GIANOLA, D. et al. Estimates of heritabilities and of genetic and phenotypic correlations among growth and reproductive traits in yearling Angus bulls. J. Anim. Sci., v. 58, p.887-893,1984.

MANUAL para exame andrológico e avaliação de sêmen animal. 2.ed. Belo Horizonte: CBRA, 1998.

MARTINEZ, M.L.; VERNEQUE, R.S.; TEODORO, R.L. et al. Correlações entre características da qualidade do sêmen e a circunferência escrotal de reprodutores da raça Gir. Rev. Bras. Zootec., v.29, p.700-706, 2000.

QUIRINO, C. R.; BERGMANN, J.A.G.; VALE FILHO, V.R. et al. Estimativa de parâmetros genéticos para características físicas do sêmen e perímetro escrotal em touros Nelore. In: ENCONTRO DE PESQUISA DA ESCOLA DE VETERINÁRIA DA UFMG, 16., 1998, Belo Horizonte. Anais... Belo Horizonte: FEPMVZ, 1998. p.34.

QUIRINO, C.R. Herdabilidades e correlações genéticas entre medições testiculares, características seminais e libido em touros Nelore. 1999. 78f. Tese (Doutorado em Ciência Animal) - Escola de Veterinária, Universidade Federal de Minas Gerais, Belo Horizonte.

VALE FILHO, V.R. Padrões de sêmen bovino, para o Brasil: análise e sugestões. In: CONGRESSO BRASILEIRO DE REPRODUÇÃO ANIMAL, 8., 1989, Belo Horizonte. Anais... Belo Horizonte: CBRA, 1989. p.94-118.

VALE FILHO, V.R.; QUIRINO, C.R.; ANDRADE, V.J. et al. Parâmetros genéticos da classificação andrológica por pontos (CAP), em touros da raça Nelore. Rev. Bras. Reprod. Anim., v.23, p.253-255, 1999. 